In Crescendo. Institucional. 2016; 7(1): 77-89

Fecha de recepción: 6 de mayo de 2016

Fecha de aceptación: 17 de mayo de 2016

\title{
MOdELO DIDÁCTICO BASADO EN LA TEORÍA DE LAS INTELIGENCIAS MÚLTIPLES PARA OPTIMIZAR EL DESARROLLO DE LA CÁTEDRA FORMACIÓN HUMANO-CRISTIANA
}

\author{
Didactic Model Based on the THEORY OF MULTIPLE \\ INTELLIGENCES TO OPTIMIZE THE DEVELOPMENT OF COURSE \\ FORMACIÓN HUMANO-CRISTIANA
}

Luis Enrique Vásquez Giménez*

\begin{abstract}
RESUMEN
$E^{1}$ objeto de la educación es el pleno desarrollo de la personalidad del estudiante, es decir, debe promover la formación integral de todas las dimensiones y capacidades que forman parte de la personalidad humana. Asimismo, debe ampliar la formación con planes y programas de estudios que den a los estudiantes la capacidad de dotarlos de conocimientos y competencias que se necesitan en el siglo XXI (Unesco, 2009). En este sentido, la presente investigación, cuyo propósito fundamental es proponer un modelo didáctico basado en la teoría de las inteligencias múltiples para optimizar el desarrollo de la cátedra Formación Humano-Cristiana (FHC) para estudiantes de pregrado de la Universidad Católica Santa Rosa (UCSAR). Este modelo didáctico constituye un valor agregado para el Vicerrectorado Académico de la universidad, orientado al desarrollo de la formación integral como eje transversal del currículo formativo de los estudiantes. Por tanto, se fundamenta en una investigación de tipo exploratoria, con un diseño de índole cuantitativo, documental y de campo. La población y muestra de estudio a considerar son nueve estudiantes egresados de las carreras de Educación, Filosofía y Comunicación Social y dos docentes que imparten la cátedra. La técnica a utilizar es la encuesta escrita con su respectivo instrumento: el cuestionario estructurado con preguntas de respuestas cerradas que restringen la respuesta a la elección entre un número limitado de categorías previamente presentadas, el cual se ha desarrollado bajo la escala de Likert. Como conclusión se señala que la optimización de la organización y desarrollo de la cátedra, desde la propuesta formulada, es una oportunidad para promover la transformación

* Aspirante al grado de doctor en Gestión y Ciencias de la Educación. Magíster en Educación con Mención en Orientación. Licenciado en Filosofía y Educación Integral. Docente e Investigador de la Red Laureate Internacional Universities (UPN-Lima).Correo electrónico: tobit7@hotmail.com.
\end{abstract}


social que se necesita mediante la investigación, el rediseño adecuado del currículo, la capacitación y el acompañamiento oportuno a los docentes, aunado a sus valores institucionales en términos de formar profesionales integrales, humana y académicamente competentes, con responsabilidad social y espíritu crítico.

Palabras clave: teorías, modelo didáctico, humano-cristiana.

\begin{abstract}
The purpose of education is the full development of the student's personality, ie, should promote the integral development of all dimensions and capabilities that are part of the human personality. It should also expand training plans and curricula that give students the ability to equip them with knowledge and skills they need in the twenty-first century (UNESCO, 2009). In this sense, this research whose primary purpose is to propose a system based on the theory of multiple intelligences to optimize the development of Human-Christian (FHC) Training lecture for undergraduate students at the Catholic University Santa Rosa Model Activity (UCSAR). This didactic model is an added value for the Academic Vice President of the University, aimed at developing comprehensive training as a central focus of the training curriculum for students. Therefore, it is based on an investigation of exploratory type. Designed with a quantitative nature, documentary and field. The study sample population and to consider are nine (9) students graduated from racing Education, Philosophy and Social Communication and two (2) teachers who teach the chair. The technique used is the Survey Written with its instrument Questionnaire structured with closed answers questions that restrict the answer to the choice between a limited number of categories previously presented, it is a Likert Scale. In conclusion it is noted that the optimization of the organization and development of the chair from the proposal is an opportunity to promote social transformation that is needed through research, proper redesign of the curriculum, training and timely support for teachers, coupled with its institutional values in terms of being comprehensive, humane and academically competent professionals with social responsibility and critical spirit.
\end{abstract}

Keywords: Theories, Teaching Model, Human-Christian.

\title{
INTRODUCCIÓN
}

\author{
La educación es sin duda el más humano y \\ humanizador de los empeños humanos \\ Fernando Savater
}

Frente a la actual crisis de valores de la sociedad contemporánea, la formación humanocristiana alcanza una validez fundamental como toma de conciencia del valor absoluto de la persona y de las normas básicas de respeto y convivencia que aseguren la dignidad de la persona y la realización de sus valores. Es así que ante la realidad social que padece nuestro pueblo, como educadores necesitamos conocer bien los compromisos que debemos asumir a fin de lograr las transformaciones sociales, políticas y económicas necesarias 
para que los individuos y la sociedad en general logren la realización justa y equitativa de todos. Por ello, cuando se habla de una formación humano-cristiana, para mejorar nuestro quehacer educativo, asumimos que esta formación implica la construcción de la persona, la reflexión, revisión y comprensión de su ser, afectividad, valores y actitudes.

En tal sentido, se indica lo importante del diseño de una propuesta de un nuevo modelo didáctico basado en la teoría de las inteligencias múltiples para optimizar el desarrollo de la cátedra Formación Humano-Cristiana, toda vez que desde el proceso formativo, del diseño y desarrollo de la cátedra se favorece dentro de un enfoque amplio y holístico que asume al estudiante de manera integral y que busca comprenderlo en un primer momento con todas sus circunstancias y ámbitos de desarrollo.

Según Gallegos (2010), los conceptos de desarrollo humano y desarrollo espiritual son diferentes, por la razón de que el primero se ubica en el nivel de la mente, de la cultura y las relaciones humanas; no implica trascendencia de la mente cognitiva y sigue basado en la idea de persona. El segundo es de nivel superior y va a la raíz de nuestra naturaleza, supera completamente la idea de humanismo y trabaja a nivel de la conciencia transpersonal. Nos habla de la unicidad, de la no-dualidad: "La inteligencia espiritual puede ser entendida también como todo lo que mantiene unidos a los seres humanos, como los valores universales, que permiten la coexistencia pacífica y el entendimiento mutuo".

Hasta la actualidad se ha investigado y hablado sobre el desarrollo de las inteligencias múltiples, inteligencia emocional, pero aún más, hoy es necesario hablar de la inteligencia espiritual. Edgar Morín (2008) plantea que justamente la misión espiritual de la educación es enseñar a que las personas se comprendan, como condición y garantía de la solidaridad intelectual y moral de la humanidad. Comprender incluye necesariamente el proceso de empatía, de identificación y de proyección.

La inteligencia espiritual se desarrolla desde la inteligencia intrapersonal (conocimiento, aprecio y comprensión de uno mismo) pasando por la inteligencia interpersonal o social (conocimiento, aprecio y comprensión de los demás) hasta llegar al conocimiento, aprecio y comprensión de todas las demás formas de vida y del propio universo. El concepto de inteligencia espiritual abre una nueva etapa en el desarrollo de la educación

Con atención a los argumentos precedentes, se hace necesario diseñar una propuesta de un nuevo modelo didáctico basado en la teoría de las inteligencias múltiples para optimizar el desarrollo de la cátedra Formación Humano-cristiana de la Universidad Católica Santa Rosa de Caracas. La propuesta busca dinamizar la misión, visión y valores institucionales, para mejorar la calidad, eficiencia y equidad del proceso de formación humana y profesional con que está comprometida, constituyéndose en la directriz que a nivel pedagógico demarca el quehacer y la unicidad de la Universidad en cuanto a su propuesta educativa. 


\section{PROBLEMA}

¿Cómo propiciar el desarrollo de la cátedra Formación Humano-cristiana en estudiantes de pregrado de la Universidad Católica Santa Rosa de Caracas, Venezuela, desde la perspectiva de la teoría de las inteligencias múltiples?

\section{OBJETIVO GENERAL}

Proponer un modelo didáctico para el desarrollo de la cátedra Formación Humanocristiana fundamentado en la teoría de las inteligencias múltiples.

\section{OBJETIVOS ESPECÍFICOS}

1. Identificar los elementos encontrados en la planificación y procedimiento del desarrollo de la cátedra Formación Humano-cristiana.

2. Organizar los elementos encontrados en el desarrollo de la planificación de la cátedra Formación Humano-cristiana.

3. Contrastar los hallazgos encontrados en el desarrollo de la cátedra Formación Humano-cristiana con la teoría propuesta.

4. Diseñar una propuesta sobre un nuevo modelo didáctico tomando como basamento la teoría de Gardner para optimizar el desarrollo de la cátedra Formación Humano-cristiana.

\section{MATERIAL Y MÉTODOS}

Si buscas resultados distintos, no hagas siempre lo mismo. Albert Einstein

El siguiente estudio se elaboró en el marco de una investigación de tipo descriptivo, el cual, según Martínez (2000, p. 8), "trata de identificar la naturaleza profunda de las realidades, su estructura dinámica, aquella que la razón plena es su comportamiento y manifestaciones". En este mismo orden de ideas, Hernández (2003, p. 220) conceptualiza como un estudio descriptivo de campo aquel que tiene como propósito "describir un evento obteniendo los datos de fuentes vivas y directas en su ambiente natural, es decir, en el contexto habitual al cual ellos pertenecen sin introducir modificaciones de ningún tipo a dicho contexto". Por consiguiente, en esta investigación se realizó la descripción de los datos obtenidos en los cuestionarios aplicados a los estudiantes y académicos de la UCSAR para verificar las inquietudes y debilidades que puedan tener sobre los conocimientos y experiencias del desarrollo de la cátedra Formación Humano-cristiana.

Otra idea relevante es la revelada por Niño Rojas (2011, p. 32): "se trata de una investigación cuyo propósito es proporcionar una visión general sobre una realidad o un aspecto de ella, de una manera tentativa o aproximativa". De acuerdo con el planteamiento conceptual acerca de la investigación descriptiva, la presente investigación se enmarcó en este nivel, por su versatilidad en la temática en la cual se conjuga en un todo. Su importancia, actualmente, se sustenta en los siguientes eventos: modelo didáctico; teoría 
de las inteligencias múltiples (inteligencia intra e interpersonal); Formación HumanoCristiana.

En atención al diseño, la investigación se clasifica en cuantitativa, documental y de campo (Arias, p. 26). Por consiguiente, la presente investigación tiene el propósito fundamental de proponer un modelo didáctico basado en la teoría de las inteligencias múltiples para optimizar el desarrollo de la cátedra Formación Humano-Cristiana para estudiantes de pregrado de la Universidad Católica Santa Rosa (UCSAR) de Caracas, Venezuela. Este modelo didáctico constituye un valor agregado para el Vicerrectorado Académico de la universidad, orientado al desarrollo de la cátedra Formación Humanocristiana integral como eje transversal del currículo formativo de los estudiantes.

En este marco, la presente exploración se canaliza en una investigación documental de las variables: modelo didáctico basado en la teoría de las inteligencias múltiples y la formación humano-cristiana. Es de acentuar que la realización de esta investigación se apoyó en fuentes bibliográficas relacionadas con las dimensiones antes mencionadas. Por ende, es una investigación documental, ya que esta se asienta en fuentes de carácter documental, es decir, en documentos de varios géneros. Aunado a lo anterior, es pertinente señalar que la delimitación de la investigación está enmarcada en un contexto real, que es la Universidad Católica Santa Rosa, Caracas, Venezuela, cuya población representativa la constituye un grupo de estudiantes egresados y docentes que imparten la cátedra. La selección de esta población de estudiantes y docentes obedece a que conforman un grupo potencial a la hora de emitir su opinión acerca del objeto de estudio de esta investigación, donde la acción de optimizar el desarrollo de la cátedra es sumamente importante para su proyección académica y profesional.

La población de estudio a considerar para esta investigación son nueve (9) estudiantes egresados de las carreras de Educación, Filosofía y Comunicación Social y dos (2) docentes que imparten la cátedra. La muestra se caracteriza por ser una población finita en la que se conoce la cantidad de unidades que la integran, y existe un registro documental de dichas unidades (Arias, 2006, p. 82), a su vez, como elemento adicional, se presta por ser una población accesible, denominada población muestreada, quiere decir, es la porción finita de la población objetivo a la que realmente se tiene acceso y de la cual se extrae una muestra representativa (Arias, 2006, p. 82). En el siguiente cuadro se detallan las características de la población y la muestra seleccionada:

Cuadro 1. Población y muestra

\begin{tabular}{|c|c|c|c|c|c|c|}
\hline \multirow[t]{2}{*}{ Población } & \multirow[t]{2}{*}{ Carreras } & \multicolumn{2}{|c|}{ Sexo } & \multirow[t]{2}{*}{ Edad } & \multirow[t]{2}{*}{ Año de egreso } & \multirow[t]{2}{*}{ Muestra } \\
\hline & & M & $\mathrm{F}$ & & & \\
\hline \multirow{3}{*}{ Egresados } & Educación & - & 3 & $25-35$ & $2010-2014$ & 9 \\
\hline & Filosofía & 2 & 1 & & & \\
\hline & Comunicación Social & 2 & 1 & & & \\
\hline Docentes & Todas & 2 & & $40-60$ & - & 2 \\
\hline Total & 3 & 6 & 5 & $25-60$ & 4 & 11 \\
\hline
\end{tabular}


La técnica a utilizar en el desarrollo de esta investigación fue la encuesta escrita (Niño Rojas, 2011, p. 63): "Entendemos por encuesta la técnica que permite la recolección de datos que proporcionan los individuos de una población, o más comúnmente de una muestra de ella, para identificar sus opiniones, apreciaciones, puntos de vista, actitudes, intereses o experiencias, entre otros aspectos. Cuando una encuesta se aplica a una fracción o muestra, se designa 'sondeo'". Se identifica por preguntas y respuestas específicas y concisas. Para la interrogación de las personas, se diseñó un cuestionario que consiste en una lista de preguntas, las cuales se les hacen a integrantes de la comunidad universitaria a encuestar, con el fin de obtener la información requerida. El instrumento de recolección de información a utilizar es el cuestionario. Niño Rojas (2011, p. 89) define el cuestionario, así: "Los cuestionarios son un conjunto de preguntas técnicamente estructuradas y ordenadas, que se presentan escritas e impresas, para ser respondidas igualmente por escrito o a veces de manera oral".

La encuesta a desarrollar es de tipo cuestionario, estructurado con 14 preguntas de respuestas cerradas que restringen la respuesta a la elección entre un número limitado de categorías previamente presentadas en el cuestionario, de esta manera (escala de Likert): 4. Totalmente de acuerdo, 3. De acuerdo, 2. Indiferente, 1. En desacuerdo.

El procesamiento utilizado para desarrollar la investigación estará enmarcado dentro de los siguientes pasos:

1. Revisión de teorías, textos, paper, revistas científicas, investigaciones y trabajos desarrollados a nivel de pregrado y postgrado referentes al tópico de la Investigación propuesta.

2. Elaboración de una matriz de variable con sus respectivas dimensiones e indicadores. En la misma, se señala la técnica e instrumento de recolección de información para cada indicador. Es de destacar, para cada indicador que se desea medir se coloca el número de ítems que los miden contemplados en el instrumento: Cuestionario.

3. Elaboración del instrumento a aplicar; es este caso, un cuestionario con escala de Likert, el cual será validado por juicios de expertos en las especialidades de pedagogía, desarrollo humano, filosofía y teología.

4. Recolección de la información a través de la aplicación del cuestionario.

5. Tabulación, análisis e interpretación de los datos obtenidos del cuestionario tanto de la muestra de egresados como de los académicos.

6. Diseño de la propuesta de modelo didáctico basado en la teoría de las inteligencias múltiples para optimizar el desarrollo de la cátedra Formación Humano-Cristiana en estudiantes de pregrado de la UCSAR.

7. Desarrollo de conclusiones y recomendaciones tomando como base a los resultados obtenidos. 


\section{RESULTADOS}

Cuando el docente llega a comprender mejor cómo se construye el saber introduce una nueva dimensión en el tratamiento y enseñanza de los contenidos disciplinares.

Habermas

La información recogida es producto de las categorías contenidas en el cuadro de operacionalización de las variables en estudio. En este orden de ideas, para el registro de datos se utilizó la estadística descriptiva y para el análisis cuantitativo se trabajó con el análisis de frecuencia y porcentajes, ya que ambos son accesibles para la organización y resumen de la información en tablas según la frecuencia con las que se presenta cada una de las variables consideradas en este estudio y cuyos datos a recolectar es a través del cuestionario aplicado a los egresados así como al personal académico de la universidad que administran la cátedra Formación Humano-Cristiana (FHC).

Los resultados obtenidos en la investigación se presentan en cuadros estadísticos que contienen una distribución absoluta y porcentual de frecuencia. Cada una está organizada en 3 columnas; en la primera aparecen las categorías; en la segunda, la frecuencia absoluta, y en la tercera, la frecuencia relativa o porcentual. La interpretación de cada categoría se realizó inmediatamente después de su presentación. Seguidamente, se presentan los resultados de la encuesta aplicada a los egresados y académicos. Es de destacar, las distribuciones de frecuencias, especialmente los datos porcentuales que se muestran gráficamente en forma de diagramas circulares y en cuadros. Los diagramas circulares son utilizados en aquellos casos donde interesa no solo mostrar el número de veces que se da una característica o atributo de manera tabular sino más bien de manera gráfica, de tal manera que se pueda visualizar mejor la proporción en que aparece esa característica respecto del total (Niño, 2011). A continuación, se pormenoriza de manera general y específica algunos de los resultados cuantitativos y cualitativos concernientes al cuestionario, tanto de los egresados (9) como del personal académico (2 profesores) que imparten la cátedra.

Tal como se evidencia en el Cuadro 4, de los once (11) encuestados, seis (6) han respondido que están de acuerdo en considerar que no hay una programación consolidada sino diversidad de contenidos, en el desarrollo de la cátedra FHC en todos los periodos

Cuadro 4. Considera que no hay una programación consolidada sino diversidad de

contenidos, en el desarrollo de la cátedra FHC en todos los periodos que se imparten.

\begin{tabular}{c|c|c}
\hline Categoría & Frecuencia & Porcentaje \\
\hline $\begin{array}{c}\text { Totalmente de } \\
\text { acuerdo }\end{array}$ & 4 & $36,4 \%$ \\
De acuerdo & 6 & $54,5 \%$ \\
Indiferente & 1 & $9,1 \%$ \\
En desacuerdo & 0 & $0,0 \%$ \\
\hline Total & $\mathbf{1 1}$ & $\mathbf{1 0 0 \%}$ \\
\hline
\end{tabular}




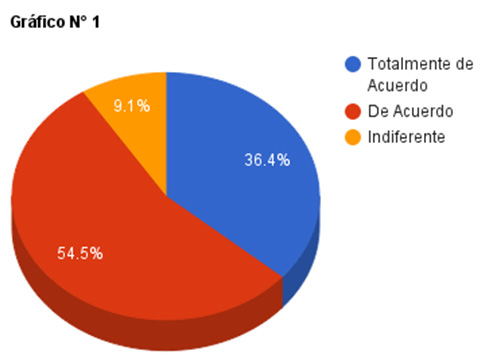

que se imparten; lo que representa el porcentaje mayor, es decir, 54,5\% como se indica en el Gráfico 1; asimismo cabe resaltar que el otro porcentaje mayor se ubica en 36,4\% en estar totalmente de acuerdo, ante tal afirmación. En este sentido, la organización de la planificación a modo de secuencias didácticas se sustentan en el reconocimiento de la necesidad de acercarse en diferentes momentos y distintas formas al conocimiento (Panigua y Umaña, 2008). Si bien es cierto que existe y se respeta la autonomía de cátedra, es necesario comprender cómo la acción formativa y en especial el proceso de aprendizaje demanda la integración y articulación de múltiples factores para lograr una formación integral, en donde se aborden las diferentes dimensiones del ser humano.

Por tanto, el profesor conceptualizado como pedagogo es aquel que es capaz de asumir exitosamente su rol de mediador de los aprendizajes a partir del dominio de la estructura de la disciplina de tal modo que pueda transformar el contenido disciplinar en contenido con fuerza pedagógica; de hacer propio el sentido de la didáctica como arte de enseñar, así como de las técnicas que le permitan poner a disposición del otro, de modo interactivo, saberes del mundo cultural, impulsar a construir nuevos significados fruto del aprendizaje compartido. En esta dinámica, se nutre y perfecciona a sí mismo a partir de la capitalización de su experiencia y práctica pedagógica transformándola en saber pedagógico (Cervantes, 2010, p. 34).

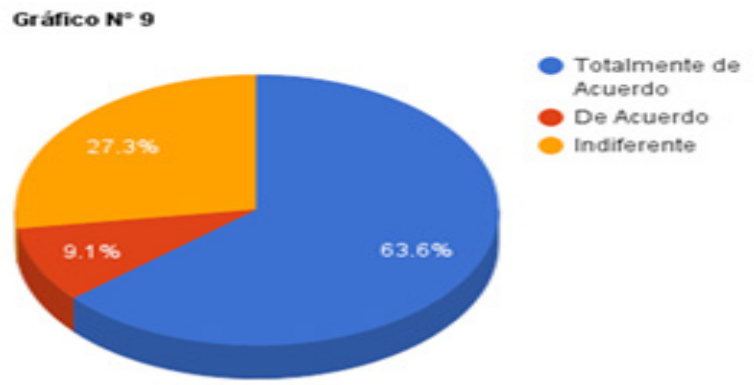

Cuadro 15. Considera que la FHC tiene que sustentarse en la misión, visión y valor es de la Universidad, que sea expresión del tipo de Universidad Católica que se quiere.

\begin{tabular}{ccc}
\hline Categoría & Frecuencia & Porcentaje \\
\hline Totalmente de & 6 & $54,5 \%$ \\
acuerdo & & \\
De acuerdo & 1 & $9,1 \%$ \\
Indiferente & 4 & $36,4 \%$ \\
En desacuerdo & 0 & $0 \%$ \\
\hline Total & $\mathbf{1 1}$ & $\mathbf{1 0 0} \%$ \\
\hline
\end{tabular}


El 63,6\% está totalmente de acuerdo en afirmar que el enfoque que debe tener la FHC en los diferentes periodos, debe ser expresión del crecimiento humano en el estudiante con miras hacia su profesión. De allí la importancia de reconocer la intencionalidad de la universidad desde su visión y misión, la cual busca responder a las necesidades de la sociedad dentro de una concepción integral del ser humano. Para lograr tal propósito, junto con la formación científica y tecnológica, que imparten las distintas facultades, ofrece al estudiante, como eje de su formación integral, la materia de FHC. En este sentido, la formación humana-cristiana, es una gran oportunidad para que el estudiante conozca y valore mejor, con el mismo rigor académico y de una forma crítica y creativa, otros saberes que le ayuden a crecer y a madurar tanto personal como profesionalmente.

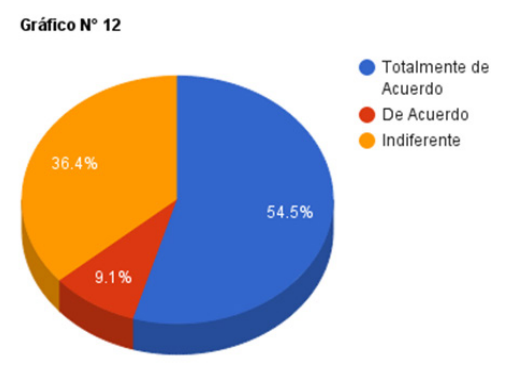

Como se puede observar en los resultados del cuadro y del gráfico, el 54,5\% de los encuestados manifiestan estar totalmente de acuerdo en considerar que la FHC tiene que sustentarse en la misión, visión y valores de la universidad, que sea expresión del tipo de universidad católica que se quiere. En consecuencia, la universidad en general y las universidades católicas, en particular, están asumiendo este reto expresado en propuestas de renovación de la concepción pedagógica, que incorporan la reflexión sobre la formación humana-cristiana en los currículos, los métodos, las prácticas, los medios de transmisión del saber y la proyección a la comunidad de referencia. De hecho, la Universidad Católica Santa Rosa es consecuente con las exigencias que se le plantean a la educación superior, desde su misión y visión, aunado a sus valores institucionales. En el pensamiento de Joyce y Weil (1985), la educación está estrechamente vinculada con los procesos de formación. La visión-misión de la universidad, pues, trasciende el componente científico, proporcionando las condiciones para que cada estudiante pueda enriquecer su proyecto de vida de una manera integral.

Cuadro 17. El desarrollo de una propuesta de un nuevo modelo didáctico basado en
la teoría de Gardner, optimizará el desarrollo de la cátedra Formación Humano-
Cristiana en estudiantes de pregrado de la Universidad Católica Santa Rosa.
\begin{tabular}{ccc} 
& \\
\hline Categoría & Frecuencia & Porcentaje \\
\hline $\begin{array}{c}\text { Totalmente de } \\
\text { acuerdo }\end{array}$ & 6 & $54,5 \%$ \\
De acuerdo & 1 & $9,1 \%$ \\
Indiferente & 3 & $27,3 \%$ \\
En desacuerdo & 1 & $9,1 \%$ \\
\hline Total & $\mathbf{1 1}$ & $\mathbf{1 0 0 \%}$ \\
\hline
\end{tabular}




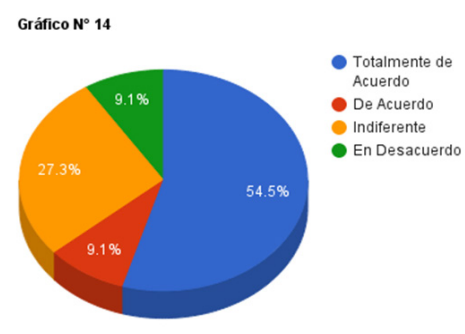

Tal como se evidencia en los resultados del cuadro y de la gráfica, el 54,5\% está totalmente de acuerdo en manifestar que una propuesta de un nuevo modelo didáctico basado en la teoría de Gardner, optimizará el desarrollo de la cátedra Formación Humano-Cristiana en estudiantes de pregrado de la Universidad Católica Santa Rosa. Toda vez que desde el proceso formativo, del diseño y desarrollo de la cátedra se favorece dentro de un enfoque amplio y holístico, que asume al estudiante de manera integral y que busca comprenderlo en un primer momento con todas sus circunstancias y ámbitos de desarrollo. Por tanto, la meta es lograr resultados efectivos y un mejoramiento de la calidad de la formación. Asimismo, reconocer la importancia que tiene la formación humano-cristiana para el desarrollo de la personalidad y para el logro de una sociedad más justa y fraterna. Buscando con ésta generar una actitud de reflexión sobre el quehacer profesional y el compromiso hacia una praxis basada en sólidos principios éticos, humanos y cristianos; tomando en consideración que dicha cátedra pretende contribuir a la difusión de los valores humanos y cristianos enfatizados desde un compromiso personal y social.

\section{DISCUSIÓN}

No hay palabra verdadera que no sea unión inquebrantable entre acción y reflexión.

Paulo Freire

Una vez tabulada la información obtenida a partir de la aplicación de los instrumentos de recolección de datos, se procedió a utilizar la técnica estadística para comprender e interpretar los resultados y así dar respuesta a las interrogantes planteadas. Dicha distribución de frecuencia corresponde al siguiente instrumento y muestras representativas: del cuestionario de escala de Lickert dirigidos a 9 egresados correspondientes a las escuelas de Educación (3), Comunicación Social (3) y Filosofía (3); asimismo, a 3 docentes que imparten la cátedra.

En tal sentido, se ha utilizado el análisis descriptivo-cuantitativo, a partir del cual se resumen los datos y características obtenidos en forma porcentual, distribuidos y agrupados en categorías, con el fin de observar el comportamiento de cada variable en la población. Asimismo, se considera cuantitativo porque el análisis de la información se efectúa con todos los datos en forma numérica (Sabino, 1992). Después de haber realizado el análisis de datos más adecuado en función a las variables estudiadas, ahora corresponde discutir 
los resultados, entre los hallazgos encontrados de la presente investigación con respecto a los estudios de autores indicados en los antecedentes de la presente investigación.

En este sentido, a partir de los hallazgos arrojados en este estudio cabe plantearse que se observa en el 90,9\% de los encuestados manifiestan estar totalmente de acuerdo en declarar que la FHC debe ser formación de la persona y debe orientarse a la creación o formación de una persona con sensibilidad humana y social. Por tanto, se pretende un enfoque integral para la formación del futuro profesional egresado de esta casa de estudios, promoviendo en el participante una formación en pro del ser humano y al desarrollo de valores auténticos que le permita ensanchar su capacidad creativa en bien de la humanidad. Por tal motivo, la formación humano-cristiana alcanza una validez fundamental como toma de conciencia del valor absoluto de la persona y de las normas básicas de respeto y convivencia que aseguren la dignidad de la persona y la realización de sus valores. Por ello, cuando se habla de una formación humano-cristiana, para mejorar nuestro quehacer educativo, se asume que esta formación implica la construcción de la persona, la reflexión, revisión y comprensión de su ser, afectividad, valores y actitudes.

El currículo formativo de la UCSAR define el marco orientador y regulador de la formación humano cristiana, académica y profesional que se imparte. Estas orientaciones asumen los principios de calidad, identidad y sustentabilidad, los cuales derivan de su misión, visión y declaración de sus valores. Estos tienden a promover la formación de profesionales con principios y valores, conocimientos y competencias que permitan un efectivo desempeño laboral. Así como su coherencia con las dimensiones que deben estar presentes en la educación a lo largo de la vida (Delors, 1996). Aprender a ser. Aprender a conocer. Aprender a hacer. Aprender a convivir.

Asimismo, es de notar el perfil del estudiante que inicia en la universidad, un joven con poca o nula práctica religiosa, o de pocas experiencias educativas cristianas, para la gran mayoría es la primera vez que escuchan hablar sobre temas religiosos o de formación humana, desarrollo personal. Al respecto, el profesor debe encontrar temas motivadores, ha de crear un clima adecuado para que el estudiante sienta interés y pueda trabajar con él y no dejando que el alumno memorice solo para aprobar. El docente, por la naturaleza propia de la cátedra, debe motivar la formación de seres autónomos, críticos, capaces de actuar en cualquier contexto y siendo capaces de vivir y formar parte de una sociedad. Todo esto no lo conseguirá si no puede captar la atención del estudiante y que este sienta interés por aquello que se le transmite.

En este sentido, (Bassedas, 1997), nos refiere que no hay ningún método perfecto, ni docentes, ni estudiantes perfectos. La perfección está en conseguir una buena compenetración y trabajar con base a los intereses del estudiante. Es necesario que el alumno vea cierta funcionalidad inmediata en los conocimientos que se le van ofertando en el aula para que este vaya integrando la nueva información con los conocimientos adquiridos previamente $y$, de esa manera, se vaya formando como un ser integral y completo y no se le vaya saturando con conocimientos banales para hacer que promocione al curso siguiente. 
Tomando en consideración que, toda vez que desde el proceso formativo, del diseño y desarrollo de la cátedra se favorezca dentro de un enfoque amplio y holístico que asuma al estudiante de manera integral y que busca comprenderlo en un primer momento con todas sus circunstancias y ámbitos de desarrollo. Por tanto, la meta es lograr resultados efectivos y un mejoramiento de la calidad de la formación. Bassedas (1997), nos aporta algunos de los criterios que son básicos en la intervención educativa o en todo momento hay que tomar en consideración todos los aspectos afectivos y relacionales que hacen posible que los estudiantes se sientan bien y tengan ganas de aprender y participar en las actividades que se llevan a cabo.

De igual manera, el presente estudio fue más allá, porque exhorta en reconocer la importancia que tiene la formación humano-cristiana para el desarrollo de la personalidad y para el logro de una sociedad más justa y fraterna. Buscando con ésta generar una actitud de reflexión sobre el quehacer profesional y el compromiso hacia una praxis basada en sólidos principios éticos, humanos y cristianos; tomando en consideración que dicha cátedra pretende contribuir a la difusión de los valores humanos y cristianos enfatizados desde un compromiso personal y social. A tal efecto, hay que considerar que el estudiante es quien construye su conocimiento, pero la actividad constructivista que realiza no es individual, sino interpersonal. En consecuencia, el docente debe intervenir precisamente en aquellas actividades que el estudiante aún no es capaz de realizar por sí mismo, pero que puede llegar a resolver si recibe ayuda pedagógicamente conveniente.

\section{CONCLUSIÓN}

Hay hombres que luchan un día y son buenos.

Hay otros que luchan un año y son mejores.

Hay quienes luchan muchos años y son muy buenos.

Pero hay los que luchan toda la vida. Esos son los imprescindibles".

Bertold Brecht

La coyuntura que se nos presenta hoy en la sociedad reclama profesionales autónomos, críticos, proactivos socialmente, capaces de trabajar en equipo y de una preocupación por aportar en la construcción de una nueva sociedad. Asimismo, requiere un profesional que sepa movilizar conocimientos de una forma profética, es decir, denunciando lo que no es conforme al bien común y a la dignidad humana. Por tanto, la Formación HumanoCristiana en el contexto actual demanda la particularidad del diálogo y el respeto por la opinión fundamentada del otro. Lo esencial no estriba en pensar igual sobre todos los temas sino precisamente que sea parte de una actitud de tolerancia, respeto y aceptación desde lo que el otro es. Por último, la optimización de la organización y desarrollo de la cátedra desde la propuesta formulada, es una oportunidad para promover la transformación generacional que se necesita mediante la investigación, el rediseño adecuado del currículo, la capacitación y el acompañamiento oportuno a los docentes. 
"La espiritualidad es la esencia de la educación. No se puede medir ni cuantificar, pero funda la acción educativa". Jacques Maritain

\section{REFERENCIAS BIBLIOGRÁFICAS}

Arias, F. (2006). El proyecto de investigación. Introducción a la metodología científica. Caracas: Episteme.

Bassedas, E. (1997). Intervención educativa y diagnóstico psicopedagógico. Barcelona: McGraw-Hill.

Cervantes, J. (2010). Encuesta sobre la percepción de las materias de Formación Humano Cristiana. Saltillo, México: Ceslas.

Delors, J. (1996). La educación encierra un tesoro. Madrid: Santillana-MEC.

Gallegos R. (2010). Educación y espiritualidad. La educación como práctica espiritual. Guadalajara: Fundación Internacional para la Educación Holista.

Hernández Sampieri, R., Fernández Collado, C., Baptista Lucio, P. (2010). Metodología de la investigación. Chile: McGraw-Hill Educación.

Joyce, B. y Weil, M. (1985). Modelos de enseñanza. Madrid: Anaya.

Niño Rojas, Víctor M. (2011). Metodología de la investigación. Bogotá: Ediciones de la U.

Martínez, M. (2000). Ciencia y arte en la metodología cualitativa. México: Trillas.

Morín, E. (2008). Los siete saberes necesarios para la educación del futuro. Unesco.

Panigua, K. y Umaña, M. (2008). La teoría de las inteligencias múltiples en la práctica docente en educación preescolar. Revista Electrónica Educare, XII. Disponible en: http://www.redalyc.org/articulo.oa?id=194114582017. Fecha de consulta: 16 de junio de 2015.

Sabino, C. (1992). El proceso de la investigación. Caracas: Panapo.

Unesco. (2009). Las nuevas dinámicas de la educación superior y de la investigación para el cambio social y el desarrollo. Conferencia Mundial sobre Educación Superior 2009. París. Disponible en: www.unc.edu.ar/institucional/noticias/2009/julio/ documentodelaconferenciamundialdeeducacion. Fecha de consulta: 16 de junio de 2015. 